\title{
Targeting GRP78 as a Basis for Enhanced Glioma Cells Killing by Anti-TfR Antibody
}

\author{
Xue WEN, Xuan CHEN, Xiaoping CHENG, Si WU \\ Hubei Polytechnic Institute, School of Nursing, Department of Basic Medical, Xiaogan, China
}

\section{ABSTRACT}

\begin{abstract}
AIM: Studies have illustrated that chemotherapeutic drugs induce endoplasmic reticulum (ER) stress in glioblastoma cells. In our previous studies, it is clear that anti-transferrin receptor (anti-TfR) monoclonal antibody (mAB) 7579 enhanced anti-tumor effects of chemotherapeutic drugs on human glioma cells in vitro. In our study, we therefore investigated how the anti-TfR antibody increased ER stress, alone or in combination with a chemotherapeutic drug.
\end{abstract}

MATERIAL and METHODS: ER stress was detected after cells were treated with anti-TfR mAb and/or Nimustine by western blot analysis. The colony survival and apoptosis of cells were detected after transfection with small interfering RNA (siRNA) and treatment with anti-TfR mAb and/or Nimustine by labeling with methylene blue and FCM.

RESULTS: The anti-TfR antibody combined with Nimustine could elevate expression of GRP78/BiP and CHOP/GADD153 and further increase ER stress. SiRNA against the ER stress marker GRP78 produced a further sensitization of glioma cells to killing by the anti-TfR antibody and/or Nimustine.

CONCLUSION: Our results show the anti-TfR antibody triggers the ER stress response. Furthermore, this anti-tumor effect is achieved via the siRNA against GRP78 for glioma cell growth and its survival. These results hold promise as a clinical approach to gene therapy for malignant gliomas.

KEYWORDS: Endoplasmic reticulum, Glioma, Glucose-regulated protein 78, Transferrin receptor

\section{INTRODUCTION}

$\mathrm{T}$

The transferrin receptor (TfR, CD71) binds the primary serum iron-transport protein, transferrin, and mediates the uptake of cellular iron (21). Even though the TfR expression in normal tissues is a relative limitation, a large number of tumor cells show high levels on their surface $(5,9,20)$. Hence, TfR could be regarded as a relevant target for antibody-based therapies against tumors. Moreover, therapeutic approaches - using anti-TfR antibodies to kill malignant cells - have been reported by many researchers (7). In previous researches, the anti-tumor effect of antibody against TfR has been demonstrated (22). Nonetheless, antiTfR mAb treatment led to an elevation in hypoxia-inducible factor-1a (HIF-1a) and HIF transcription targets, which might bring about tumor angiogenesis and enhance tumor progression and metastasis (6).

The unfolded protein response (UPR), namely, the endoplasmic reticulum (ER) stress response, is a series of evolutionarily conserved signaling pathways intending to restore homeostasis under circumstances of ER stress (18). Glucoseregulated protein $78(\mathrm{GRP} 78)$ referred to as $\mathrm{BiP}$ functions as a central regulator of ER owing to its roles in protein folding and assembly, degradation of misfolded protein, ER $\mathrm{Ca}\left(2^{+}\right)$binding and activation control of trans-membrane ER stress sensors (12). C/EBP homologous protein (CHOP), one of components of the ER stress-mediated apoptosis pathway, is known as growth arrest and DNA damage-inducible gene 153 (GADD153)(13), while CHOP/GADD153 is a key molecule in 
ER stress-mediated apoptosis and diseases. Under intense or persistently high stress, the defensive functions (e.g. elevated levels of GRP78) are overwhelmed and the pro-apoptotic components (e.g. CHOP and caspase-4) become dominant and cause cell death (8).

Malignant gliomas have high resistance to chemotherapy and radiotherapy and elimination of residual tumor cells, which is still a tremendous challenge. Studies have shown that chemotherapeutic drugs induce CHOP and GRP78 in glioblastoma cell lines (17). Our hypothesis was that the antiTfR antibody would increase ER stress. In this report, we show that this is indeed the case. SiRNA against GRP78 further inhibited the growth of glioma cells by the anti-TfR antibody and/or Nimustine. Results mentioned above suggest GRP78 is a vital targeted gene therapy for malignant gliomas.

\section{MATERIAL and METHODS}

\section{Cells, Antibodies and Chemotherapeutic Drugs}

U251 and U87MG cells were from the Shanghai Institute for Biological Science of the Chinese Academy of Science, and anti-TfR mAb 7579 was purified and characterized in our laboratory as described previously (22). The antibodies against GRP78, CHOP, actin and the secondary antibodies coupled to horseradish peroxidase were obtained from Santa Cruz Biotechnology (Santa Cruz, CA, USA), while chemotherapeutic Nimustine was bought from Daiichi Sankyo (Japan). Anti-TfR mAb and Nimustine effects on these cells have been published elsewhere (22).

\section{Western Blot Analysis}

It took 72 hours to treat U87MG and U251 cells with anti-TfR $\mathrm{mAb}(0,50,100,200$, and $300 \mu \mathrm{g} / \mathrm{mL})$ and/or Nimustine $(0$, $20,40,60$, and $80 \mu \mathrm{g} / \mathrm{mL}$ ). Based on western blot analysis, GRP78 or CHOP expression in cells were evaluated with antiGRP78 or anti-CHOP antibodies as major antibody, followed by secondary horseradish peroxidase (HRP)-conjugated goat anti-mouse IgG antibodies according to manufacturer's recommendations (2).

\section{Transfections with SiRNA}

We transfected siRNA against GRP78 with LipofectAMINE 2000 (Invitrogen, San Diego, CA) into U251 and U87MG cells in line with the manufacturer's instructions. The siRNAs sequences reflect the following aspects: siRNA against green fluorescent protein (si-GFP) was used as a control, 5'-CAAGCUGACCCUGAAGUUCTT-3' (sense) and 5' -GAACUUCAGGGUCAGCUUGTT-3' (antisense); siGRP78, 5'-GGAGCGCAUUGAUACUAGATT-3' (sense) and 5 '-UCUAGUAUCAAUGCGCUCCTT-3' (antisense) (14). Transfected cells were counted and detected by western blot analysis to check the expression of GRP78.

\section{Colony Formation Assays}

After transfection with siRNA six-well plates at 200 cells per well were used to plant U87MG and U251 cells which were allowed to adhere overnight. Then, the medium was changed to include anti-TfR mAb $(0,50,100,200$, and $300 \mu \mathrm{g} / \mathrm{mL})$ and/ or Nimustine $(0,20,40,60$, and $80 \mu \mathrm{g} / \mathrm{mL})$ for 48 hours. Cells were incubated in fresh medium for 14 days without drug. With $1 \%$ methylene blue in methanol for 4 hours, colonies were visualized by their stain and quantified as well after termination of assay (2).

\section{Cell Apoptosis Analysis}

U87MG and U251 cells $\left(2 \times 10^{5}\right)$ were planted in six-well plates after transfecting with siRNA for 24 hours. Subsequently, these cells were co-cultured with anti-TfR mAb $(0,50,100$, 200 , and $300 \mu \mathrm{g} / \mathrm{mL}$ ) and/or Nimustine $(0,20,40,60$, and 80 $\mu \mathrm{g} / \mathrm{mL}$ ) for 72 hours. The glioma cells' apoptosis was detected by flow cytometry with FITC-Annexin V and PI according to the manufacturer's recommendations.

\section{Statistical Analysis}

Figures were analyzed by Student's t-test in a statistical way or analysis of variance (ANOVA). $\mathrm{p}<0.05$ suggested statistical significance.

\section{RESULTS}

\section{Anti-TfR mAb Treatment of Glioma Cells Triggers CHOP/ GADD153 and GRP78}

After U251 or U87MG cells were treated with rising concentrations on anti-TfR mAb and/or Nimustine, the expressions of GRP78 and CHOP in cells were detected by western blot analysis to determine the ability of anti-TfR mAb and/or Nimustine to induce the ER stress response (ESR) in glioma cells. As shown in Figure 1A-C, not only Nimustine but also anti-TfR mAb caused increased expression of GRP78 and $\mathrm{CHOP}$, and a striking induction of GRP78 and CHOP was observed when Nimustine and anti-TfR mAb were combined. These data indicated that the anti-TfR mAb is a fresh inducer for the response to ER stress.

\section{Inhibition Growth of U87MG and U251 Cells by GRP78 Knockdown}

The GRP78 expression in U87MG as well as U251 cells was significantly reduced by si-GRP78 (data not shown).

\section{Knockdown of GRP78 Sensitizes Gliomas to Anti-TfR mAb and/or Nimustine}

To explore whether glioma cells increased the expression of ER chaperone GRP78 for protection against anti-TfR mAb and/ or Nimustine-mediated cell death, we transfected U251 and U87MG cells with siRNA against GRP78. We used increasing concentrations of anti-TfR mAb and/or Nimustine to detect the drug sensitivity of the cells after GRP78 knockdown (Figure 2A-C). Results by colony survival assays indicated GRP78 knockdown remarkably sensitized glioma cells to antiTfR mAb and/or Nimustine, which suggested GRP78 might be a protector against the cytotoxic effects of anti-TfR mAb and Nimustine.

SiGRP78 Transfection Tumor Cells Apoptosis Induced by Anti-TfR $\mathrm{mAb}$ and/or Nimustine in vitro

When GRP78 knockdown cells were treated with a range (20$80 \mu \mathrm{g} / \mathrm{mL}$ ) of Nimustine concentrations for 72 hours, there was 


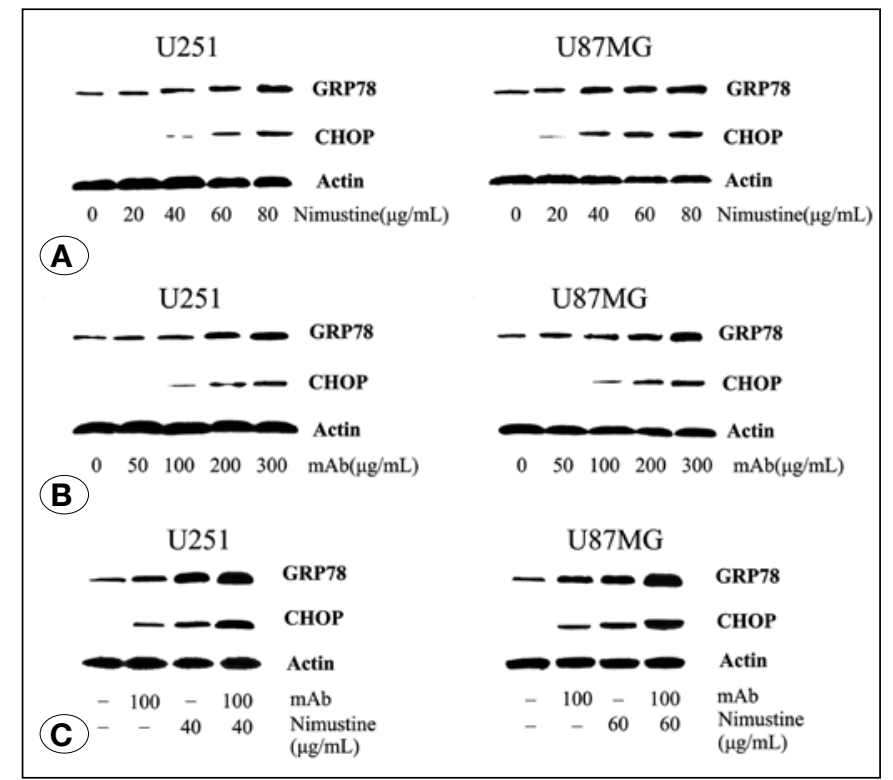

Figure 1: Nimustine and/or anti-TfR $m A b$ induced the expression of GRP78 and CHOP and in glioma cells.

A) U87MG and U251 cells were treated with Nimustine $(0,20,40$, 60 , and $80 \mu \mathrm{g} / \mathrm{mL}$ ). GRP78 and CHOP expression were detected by western blot. B) U251 and U87MG cells were treated with anti-TfR mAb (0, 50, 100, 200, and $300 \mu \mathrm{g} / \mathrm{mL}) \mathrm{GRP} 78$ and CHOP expression were detected by western blot. C) U251 and U87MG cells were treated with anti-TfR $\mathrm{mAb}(100 \mu \mathrm{g} / \mathrm{mL})$ and/or Nimustine $(40 \mu \mathrm{g} / \mathrm{mL})$, and U87MG cells were treated with anti-TfR $\mathrm{mAb}$ $(100 \mu \mathrm{g} / \mathrm{mL})$ and/or Nimustine $(60 \mu \mathrm{g} / \mathrm{mL})$. GRP78 and CHOP expression were detected by western blot.

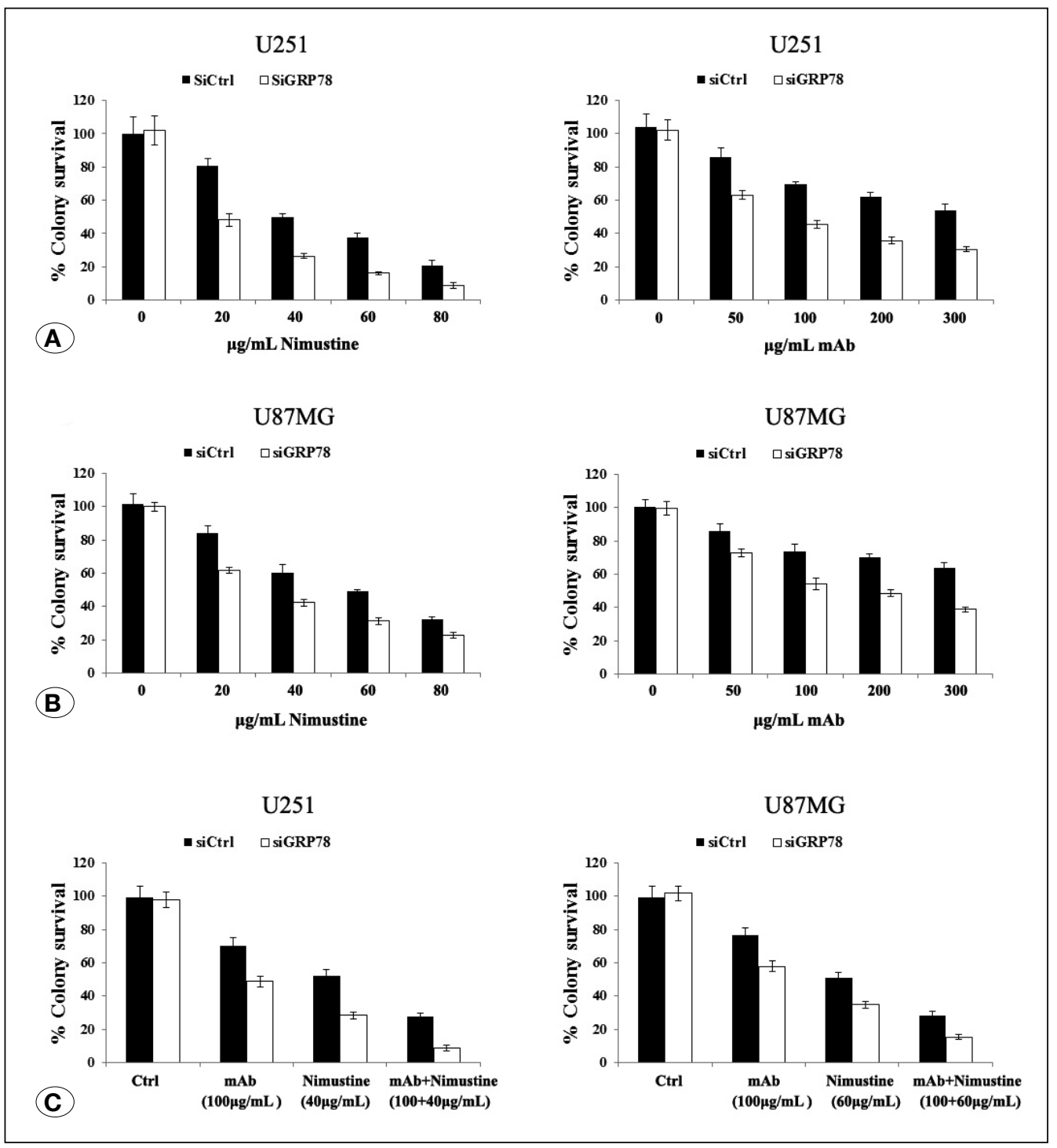

Figure 2: Knockdown of GRP78 sensitizes glioma cells to anti-TfR mAb and/or Nimustine.

A) U251 cells were seeded in six-well plates at 200 cells per well after transfection with siGRP78 or si-GFP for 24 hours. Subsequently, the cells were treated with Nimustine $(0,20$, 40,60 , and $80 \mu \mathrm{g} / \mathrm{mL}$ ) or antiTfR mAb $(0,50,100,200$, and $300 \mu \mathrm{g} / \mathrm{mL}$ ) for 48 hours. The percent of colony survival was measured by methylene blue staining. B) U87MG cells were seeded in six-well plates at 200 cells per well after transfection with si-GRP78 or si-GFP for 24 hours. Subsequently, the cells were treated with Nimustine (0, $20,40,60$, and $80 \mu \mathrm{g} / \mathrm{mL})$ or anti-TfR mAb (0, 50, 100, 200, and $300 \mu \mathrm{g} / \mathrm{mL}$ ) for 48 hours. The percent of colony survival was measured by methylene blue staining. C) U251 and U87MG cells were seeded in six-well plates at 200 cells per well after transfection with si-GRP78 or si-GFP for 24 hours. Subsequently, the cells were treated with Nimustine (U251, $40 \mu \mathrm{g} / \mathrm{mL}$ and U87MG $60 \mu \mathrm{g} / \mathrm{mL}$ ) and/or anti-TfR $\mathrm{mAb}(100 \mu \mathrm{g} / \mathrm{mL})$ for 48 hours. The percent of colony survival was measured by methylene blue staining. Each group was performed in triplicate $(p<0.05)$. 
an obvious growth in the ratio of apoptotic cells (Figure 3AC). Meanwhile, the percentage of cells undergoing apoptosis increased in a dose-dependent manner. In addition, anti-TfR $\mathrm{mAb}$ also had an increase in the percentage of apoptotic cells in a dose-dependent manner. When Nimustine and anti-TfR
mAb were used as a combination, more GRP78 knockdown cells experienced apparent apoptosis. These results confirmed that downregulation of GRP78 could be an effective approach to induce drug sensitivity in glioma cells.

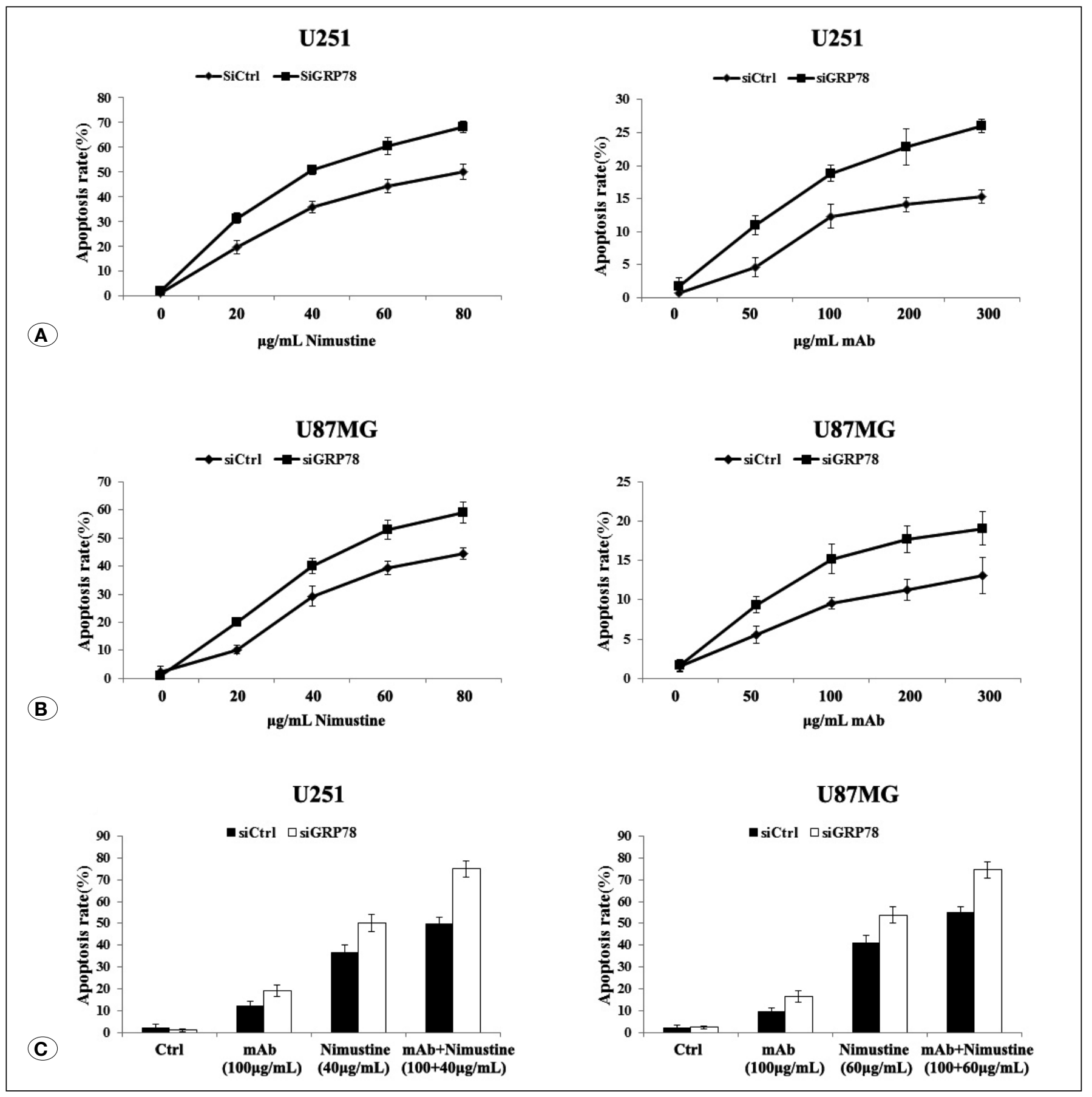

Figure 3: SiGRP78 transfection tumor cells apoptosis induced by anti-TfR mAb and/or Nimustine in vitro.

A) Apoptosis of U251 cells were measured by flow cytometry after transfected with si-GRP78 or si-GFP and treated with the indicated concentration of Nimustine or anti-TfR mAb. B) Apoptosis of U87MG cells was measured by flow cytometry after transfected with siRNA against GRP78 or GFP and treated with the indicated concentration of Nimustine or anti-TfR mAb. C) Apoptosis of U251 and U87MG cells was measured by flow cytometry after transfected with siRNA against GRP78 or GFP and treated with the indicated concentration of Nimustine and anti-TfR mAb. Each group was performed in triplicate $(p<0.05)$. 


\section{DISCUSSION}

Recent findings show that ER stress plays a critical role in cancer progression $(1,12,16,23)$. Although the discovery of ER stress in tumor growth contributes to the development of latest targeted therapeutic approaches against cancer (12), we know little on the potential exploitation in drug resistance and cancer therapy. In previous studies, we have illustrated an anti-TfR $\mathrm{mAb}$ that could inhibit cell proliferation, induce cell apoptosis, and enhance cytotoxic effects of chemotherapeutic drugs on glioma cells. We therefore investigated that whether the anti-TfR antibody alone or the combination with Nimustine would lead to the increasing of ER stress with tumor cell death.

We detected ER stress by two overexpression components of the ESR: One is a pro-apoptotic constituent and transcription factor of the ESR, known as CHOP/GADD153 (1), and the other one is an essential element of the protective arm and ER chaperone of ESR, known as GRP78/BiP (12). An increased induction of GRP78 was detected in a dose-dependent manner and $\mathrm{CHOP}$ induction was generally observed and increased in two glioma cell lines with anti-TfR mAb or Nimustine (Figure $1 \mathrm{~A}-\mathrm{C})$. The glioma cells resulted from severe induction of CHOP and GRP78 after the combination treatment of anti$\mathrm{TfR} \mathrm{mAb}$ and Nimustine, implying the existence of strong ER stress.

Taking into account that stress induction of GRP78 represents protection to tumor and confers chemoresistance, knockdown of GRP78 may be an effective target for anticancer therapy. Results show that both glioma cell lines witnessed significant increases in chemosensitivity to anti-TfR mAb and/or Nimustine (Figure 2A-C). Recent studies demonstrate that blocking GRP78 expression by small molecules may represent the latest approach in future cancer therapy $(3,4,10,14,15)$. Silencing GRP78 experiments were done to explore whether gliomas rely on GRP78 for protection against anti-TfR mAbmediated cell death. Our results show that glioma cells as GRP78 knockdown by siRNA exhibited increased cell apoptosis when treated with anti-TfR mAb or Nimustine and a higher cell apoptosis rate was observed when the combination was used (Figure 3A-C)

TfR is expressed not only at the brain cell membrane (BCM) but also at the blood-brain barrier (BBB)(11), and research shows that $\mathrm{TfR}$ against the rat $\mathrm{TfR}$ undergoes a PNA radiopharmaceutical through the BBB in vivo (19). These studies imply that the anti-TfR mAb could deliver a siRNA against GRP78 and/or chemotherapeutic drugs to experimental gliomas.

\section{- CONCLUSION}

Our results suggest that anti-TfR antibody could elevate expression of CHOP and GRP78 and further increase ER stress when combined with Nimustine. GRP78, one of the protective ER markers, knockdowns further sensitized glioma cells to killing by the anti-TfR antibody, Nimustine or the combination. Overall, our results present a target for increasing drug resistance.

\section{ACKNOWLEDGEMENT}

This work was supported by Hubei Province health and family planning scientific research project (NO.WJ2017Q040 to Xue Wen).

\section{REFERENCES}

1. Boyce M, Yuan J: Cellular response to endoplasmic reticulum stress: A matter of life or death. Cell Death Differ 13:363-373, 2006

2. Cho HY, Wang W, Jhaveri N, Torres S, Tseng J, Leong MN, Lee DJ, Goldkorn A, Xu T, Petasis NA, Louie SG, Schonthal AH, Hofman FM, Chen TC: Perillyl alcohol for the treatment of temozolomide-resistant gliomas. Mol Cancer Ther 11:24622472, 2012

3. Deng WG, Ruan KH, Du M, Saunders MA, Wu KK: Aspirin and salicylate bind to immunoglobulin heavy chain binding protein (BiP) and inhibit its ATPase activity in human fibroblasts. Faseb J 15:2463-2470, 2001

4. Ermakova SP, Kang BS, Choi BY, Choi HS, Schuster TF, Ma WY, Bode AM, Dong Z: (-)-Epigallocatechin gallate overcomes resistance to etoposide-induced cell death by targeting the molecular chaperone glucose-regulated protein 78 . Cancer Res 66:9260-9269, 2006

5. Gatter KC, Brown G, Trowbridge IS, Woolston RE, Mason DY: Transferrin receptors in human tissues: Their distribution and possible clinical relevance. J Clin Pathol 36:539-545, 1983

6. Hong Y, Yang J, Shen X, Zhu H, Sun X, Wen X, Bian J, Hu $H$, Yuan L, Tao J, Lei P, Shen G: Sinomenine hydrochloride enhancement of the inhibitory effects of anti-transferrin receptor antibody-dependent on the COX-2 pathway in human hepatoma cells. Cancer Immunol Immunother 62:447454, 2012

7. Jones DT, Trowbridge IS, Harris AL: Effects of transferrin receptor blockade on cancer cell proliferation and hypoxiainducible factor function and their differential regulation by ascorbate. Cancer Res 66:2749-2756, 2006

8. Kardosh A, Golden EB, Pyrko P, Uddin J, Hofman FM, Chen TC, Louie SG, Petasis NA, Schonthal AH: Aggravated endoplasmic reticulum stress as a basis for enhanced glioblastoma cell killing by bortezomib in combination with celecoxib or its non-coxib analogue, 2,5-dimethyl-celecoxib. Cancer Res 68:843-851, 2008

9. Keer HN, Kozlowski JM, Tsai YC, Lee C, McEwan RN, Grayhack JT: Elevated transferrin receptor content in human prostate cancer cell lines assessed in vitro and in vivo. J Urol 143:381-385, 1990

10. Lee AS: GRP78 induction in cancer: Therapeutic and prognostic implications. Cancer Res 67:3496-3499, 2007

11. Lee HJ, Boado RJ, Braasch DA, Corey DR, Pardridge WM: Imaging gene expression in the brain in vivo in a transgenic mouse model of Huntington's disease with an antisense radiopharmaceutical and drug-targeting technology. $\mathrm{J}$ Nucl Med 43:948-956, 2002

12. Li J, Lee AS: Stress induction of GRP78/BiP and its role in cancer. Curr Mol Med 6:45-54, 2006 
13. Oyadomari S, Mori M: Roles of CHOP/GADD153 in endoplasmic reticulum stress. Cell Death Differ 11:381-389, 2004

14. Park HR, Ryoo IJ, Choo SJ, Hwang JH, Kim JY, Cha MR, Shin-Ya K, Yoo ID: Glucose-deprived HT-29 human colon carcinoma cells are sensitive to verrucosidin as a GRP78 down-regulator. Toxicology 229:253-261, 2007

15. Park HR, Tomida A, Sato S, Tsukumo Y, Yun J, Yamori T, Hayakawa Y, Tsuruo T, Shin-Ya K: Effect on tumor cells of blocking survival response to glucose deprivation. J Natl Cancer Inst 96:1300-1310, 2004

16. Pyrko P, Kardosh A, Liu YT, Soriano N, Xiong W, Chow RH, Uddin J, Petasis NA, Mircheff AK, Farley RA, Louie SG, Chen TC, Schonthal AH: Calcium-activated endoplasmic reticulum stress as a major component of tumor cell death induced by 2,5-dimethyl-celecoxib, a non-coxib analogue of celecoxib. Mol Cancer Ther 6:1262-1275, 2007

17. Pyrko P, Schonthal AH, Hofman FM, Chen TC, Lee AS: The unfolded protein response regulator GRP78/BiP as a novel target for increasing chemosensitivity in malignant gliomas. Cancer Res 67:9809-9816, 2007
18. Rana T, Shinde VM, Starr CR, Kruglov AA, Boitet ER, Kotla $P$, Zolotukhin S, Gross AK, Gorbatyuk MS: An activated unfolded protein response promotes retinal degeneration and triggers an inflammatory response in the mouse retina. Cell Death Dis 5:e1578, 2014

19. Shi N, Boado RJ, Pardridge WM: Antisense imaging of gene expression in the brain in vivo. Proc Natl Acad Sci USA 97:14709-14714, 2000

20. Shindelman JE, Ortmeyer AE, Sussman HH: Demonstration of the transferrin receptor in human breast cancer tissue. Potential marker for identifying dividing cells. Int $\mathrm{J}$ Cancer 27:329-334, 1981

21. Trowbridge IS, Shackelford DA: Structure and function of transferrin receptors and their relationship to cell growth. Biochem Soc Symp 51:117-129, 1986

22. Wen X, Cheng X, Hu D, Li W, Ha J, Kang Z, Zhang M, Huang $Y$, Wu S: Combination of curcumin with an anti-transferrin receptor antibody suppressed the growth of malignant gliomas in vitro. Turk Neurosurg 26: 209-214, 2016

23. Wu J, Kaufman RJ: From acute ER stress to physiological roles of the unfolded protein response. Cell Death Differ 13:374-384, 2006 